\title{
On the eigenfunction expansion for Hamilton operators
}

\author{
Alexander Komech ${ }^{1}$ and Elena Kopylova ${ }^{2}$
}

\begin{abstract}
A spectral representation for solutions to linear Hamilton equations with nonnegative energy in Hilbert spaces is obtained. This paper continues our previous work on Hamilton equations with positive definite energy. Our approach is a special version of M. Krein's spectral theory of $J$-selfadjoint operators in Hilbert spaces with indefinite metric.

As a principal application of these results, we justify the eigenfunction expansion for linearized nonlinear relativistic Ginzburg-Landau equations.
\end{abstract}

Mathematics Subject Classification (2010). 35P, 37K.

Keywords. Hamilton equation; selfadjoint operator, $J$-selfadjoint operator, Krein space, spectral resolution, spectral representation, secular solutions, eigenvector, Jordan block, Ginzburg-Landau equation, kink, asymptotic stability, generalized eigenfunction, eigenfunction expansion, Fermi golden rule.

\section{Contents}

1 Introduction . . . . . . . . . . . . . . . . . . . . . . . 332

2 Reduction to symmetric generator . . . . . . . . . . . . . 335

3 Dynamical group and canonical form . . . . . . . . . . . . . 340

4 Application to eigenfunction expansion . . . . . . . . . . . . . 344

5 Orthogonal eigenfunction expansion . . . . . . . . . . . . . . 346

6 Nonorthogonal eigenfunction expansion . . . . . . . . . . . . 350

7 Symplectic normalization . . . . . . . . . . . . . . 354

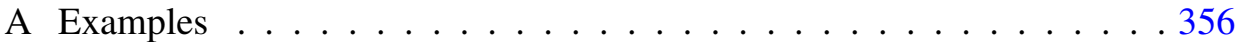

References . . . . . . . . . . . . . . . . . . 357

${ }^{1}$ The first author was supported partly by Alexander von Humboldt Research Award, Austrian Science Fund (FWF): P22198-N13, and the Russian Foundation for Basic Research.

2 The second author was supported partly by Austrian Science Fund (FWF): M1329-N13, and the Russian Foundation for Basic Research. 


\section{Introduction}

We consider complex linear Hamilton operators in a complex Hilbert space $\mathcal{X}$,

$$
A=J B, \quad \text { where } B^{*}=B, J^{*}=-J, J^{2}=-1 .
$$

In particular, the operator $J: X \rightarrow X$ is bounded. The selfadjoint operator $B$ is defined on a dense domain $D(B) \subset \mathcal{X}$. Our aim is to prove the well-posedness of the Cauchy problem for the equation

$$
\dot{X}(t)=A X(t),
$$

and obtain a spectral representation for solutions and the corresponding spectral resolution for $A$. E.g., for $J=i$ the solutions are given by $X(t)=e^{i B t} X(0)$. A more general 'commutative case', when $J B=B J$, reduces to $J=i$, since $J B=i B_{1}$, where $B_{1}=-i J B$ is the selfadjoint operator. However, $J B \neq B J$ for linearizations of $U(1)$-invariant nonlinear Schrödinger equations as shown in Appendix of [26].

We develop the theory in the case of nonnegative 'energy operators' $B$ with spectral gap and finite 'degeneracy of the vacuum':

Condition I. $\quad \sigma(B) \subset\{0\} \cup[\delta, \infty), \quad \delta>0$,

Condition II. $\operatorname{dim} \operatorname{Ker} B<\infty$.

These conditions hold, in particular, for all equations considered in [13] and [21]-[23]. The motivation for the theory was discussed in [26], in which the simplest case $\sigma(B) \subset(\delta, \infty)$ (i.e., $\operatorname{dim} \operatorname{Ker} B=0$ ) was studied.

We reduce the problem to a selfadjoint generator developing a special version of M. Krein's spectral theory of $J$-selfadjoint operators in Hilbert spaces with indefinite metric [2], [27]. We apply this version for justification of the eigenfunction expansions for the linearization of relativistic nonlinear Ginzburg-Landau equation [22]. The generator of the linearization reads

$$
A=\left(\begin{array}{cc}
0 & 1 \\
-S & 0
\end{array}\right)
$$

where

$$
S:=-\frac{d^{2}}{d x^{2}}+m^{2}+V_{0}(x) .
$$

Our results are concerned with the following. 
- The existence and uniqueness and formula for generalized solutions to (1.2) under conditions (1.3) and (1.4) for all initial states $X$ with finite energy $\langle B X, X\rangle$. Here, $\langle\cdot, \cdot\rangle$ stands for the scalar product in $X$.

- The eigenfunction expansion

$$
\left(\begin{array}{l}
\psi(t) \\
\dot{\psi}(t)
\end{array}\right)=t \Phi_{0}+\Psi_{0}+\sum e^{-i \omega_{k} t} C_{k} a_{k}+\int_{|\omega| \geq m} e^{-i \omega t} C(\omega) a_{\omega} d \omega
$$

for solutions to (1.2) with generator (1.5). Here, $\Phi_{0} \in \operatorname{Ker} A$, and $a_{k}$ are the eigenvectors of $A, \Psi_{0}$ is the associated eigenvector to $\Phi_{0}$, while $a_{\omega}$ are generalized eigenfunctions of $A$.

Such eigenfunction expansions were used in $[4,5,22]$ for the calculation of 'Fermi Golden Rule' (FGR) in the context of the nonlinear Schrödinger and KleinGordon equations. This is a nondegeneracy condition, which was introduced in [42] in the framework of nonlinear wave and Schrödinger equations. This condition means a strong coupling of discrete and continuous spectral components of solutions providing the radiation of energy to infinity and which results in the asymptotic stability of solitary waves. The calculation of FGR, as given in $[4,5,26]$, relies on eigenfunction expansions of type (1.6). Our main Theorem 6.4 justifies the eigenfunction expansion [22, (5.14)], for which no detailed proof was given before. This justification was one of our main motivation for writing the present paper.

The eigenfunction expansion (1.6) extends our previous result [26], where the expansion was established only for odd solutions. In this framework we have Ker $B=0$ and $\Phi_{0}=\Psi_{0}=0$. This framework was sufficient for the proof of asymptotic stability of standing solitons for the nonlinear relativistic GinzburgLandau equations under odd perturbations [22]. However, to establish the asymptotic stability under arbitrary perturbations we need the expansion (1.6) for solutions without antisymmetry.

Let us comment on our approach. First, we reduce the abstract problem (1.2) under conditions (1.3) and (1.4) to a selfadjoint generator justifying the classical M. Krein transformation [10]. This reduction is a special version of spectral theory of $J$-selfadjoint operators in Hilbert spaces with indefinite metric [2, 27], extending our approach [26] to the case $\operatorname{Ker} B \neq 0$. This extension required new robust ideas i) to analyze the structure of spectrum of the reduced selfadjoint operator, and ii) to find the canonical form of the Hamilton operator. We provide a broad range of examples satisfying all the imposed conditions (1.3), (1.4), (2.12), and (3.1). 
Second, we apply this abstract spectral theory to operator (1.5) and construct the eigenfunction expansion for the reduced selfadjoint operator following the method of Section 5 from [26]. At last, we deduce (1.6) by extending our approach from [26], which relies on the methods of PDO.

One of our novelties is a vector-valued treatment of the convergence of the integral over the continuous spectrum in (1.6). Namely, we show that the integral is the limit of the corresponding integrals over $m \leq|\omega| \leq M$ as $M \rightarrow \infty$ in the Sobolev space $H^{1}(\mathbb{R})$. In its own turn, the integral over $m \leq|\omega| \leq M$ is absolutely converging in the weighted $L^{2}$-space with the weight $(1+|x|)^{-s}$, where $s>1$.

Finally, calculation of the symplectic normalization of the generalized eigenfunctions requires extra arguments pertaining to the nondegenerate case [26].

We now give some comments on the related works. Some spectral properties of the Hamilton non-selfadjoint operators were studied by V. Buslaev and G. Perelman [3, 4, 5], M. B. Erdogan and W. Schlag [8, 39], S. Cuccagna, D. Pelinovsky and V. Vougalter [7]. It is worth noting that the eigenfunction expansions of $J$-selfadjoint operators were not justified previously.

Spectral resolution of bounded $J$-selfadjoint nonnegative operators in Krein spaces was constructed by M. Krein, H. Langer and Yu. Shmul'yan [27, 28], and extended to unbounded definitizable operators by M. Krein, P. Jonas, H. Langer and others $[14,15,17,29,30]$. The corresponding unitary operators were examined by P. Jonas [16]. However, the spectral resolution alone is insufficient for justification of eigenfunction expansion. Our version of the theory under conditions (1.3), and (1.4) allows us to justify the eigenfunction expansion (1.5).

The spectral theory of definitizable operators was applied to the Klein-Gordon equations with non-positive energy by P. Jonas, H. Langer, B. Najman and C. Tretter $[18,19,31,32,33]$, where the existence and uniqueness of classical solutions were proved, and the existence of unstable eigenvalues (imaginary frequencies) was studied. The instability is related to the known Klein paradox in quantum mechanics [38].

The scattering theory for the Klein-Gordon equations with non-positive energy was developed by C. Gérard and T. Kako using the theory of definitizable operators in Krein spaces [9, 20].

The plan of our paper is as follows. In Section 2, we justify the M. Krein transformation under conditions (1.3) and (1.4), and find the structure of spectrum of the corresponding selfadjoint generator. In Section 3, we construct the spectral representation for solutions to (1.2) and deduce the canonical form of the Hamilton generator. In Section 4, we check all conditions (1.3), (1.4), (2.12), and (3.1) for 
operator (1.5). In Sections 5 and 6, we justify the eigenfunction expansion (1.6) by applying the methods of Sections 3-4. In Section 7, we calculate symplectic normalization of the generalized eigenfunctions. Finally, in the Appendix we construct examples of Hamilton equations satisfying all the imposed conditions.

Acknowledgments. The authors take pleasure in thanking A. Kostenko and G. Teschl for useful discussions on spectral theory of $J$-selfadjoint operators.

\section{Reduction to symmetric generator}

In this section, we shall reduce (1.2) to an equation with selfadjoint generator.

2.1. Generalized solutions. Throughout the paper, $D(B)$ is a dense domain of the selfadjoint operator $B$. We set

$$
\Lambda:=B^{1 / 2} \geq 0
$$

and denote by $\mathcal{V} \subset \mathcal{X}$ the Hilbert space which is the domain of $\Lambda$ endowed with the norm

$$
\|X\|_{\mathcal{v}}:=\|\Lambda X\|_{x}+\|X\|_{x} .
$$

We have the continuous injections of Hilbert spaces $\mathcal{V} \subset \mathcal{X}$, and the operator

$$
\Lambda: v \longrightarrow X
$$

is continuous. For example, $\mathcal{V}$ becomes the Sobolev space $H^{1}\left(\mathbb{R}^{n}\right)$ if $X=L^{2}\left(\mathbb{R}^{n}\right)$ and $A=-i \Delta$.

Since $\Lambda$ and $B$ are selfadjoint operators, we have

$$
\mathcal{X}=\mathcal{K} \oplus \mathcal{R}, \quad \mathcal{K}:=\operatorname{Ker} \Lambda=\operatorname{Ker} B, \quad \mathcal{R}:=\operatorname{Ran} \Lambda=\operatorname{Ran} B=\mathcal{K}^{\perp} .
$$

By definition (2.1),

$$
\mathcal{K} \subset \mathcal{V} .
$$

Further, we assume henceforth that $\mathcal{R}$ is endowed with the norm of $X$. Then

$$
\Lambda_{+}:=\left.\Lambda\right|_{\mathcal{R}}: \mathcal{R} \cap \mathcal{V} \longrightarrow \mathcal{R}
$$

is an invertible operator by (1.3); i.e.,

$$
\Lambda_{+}^{-1}: \mathcal{R} \longrightarrow \mathcal{V}
$$


is the bounded operator. We will consider solutions

$$
X(t) \in C(\mathbb{R}, \mathcal{V})
$$

to equation (1.2). The equation will be understood in the sense of mild solutions [6]

$$
X(t)-X(0)=A \int_{0}^{t} X(s) d s, \quad t \in \mathbb{R},
$$

where the Riemann integral converges in $\mathcal{V}$ by (2.6).

2.2. Krein substitution. Let us reduce equation (2.7) by the well-known substitution

$$
Z(t):=\Lambda X(t) \in C(\mathbb{R}, \mathcal{R})
$$

used by M. Krein in the theory of parametric resonance: see formula (1.40) of [10, Chapter VI]. Applying $\Lambda$ to both sides of equation (2.7), we obtain

$$
Z(t)-Z(0)=\Lambda J \Lambda \int_{0}^{t} Z(s) d s, \quad t \in \mathbb{R} .
$$

Formally, (2.9) reads

$$
i \dot{Z}(t)=H Z(t), \quad t \in \mathbb{R},
$$

where $H$ stands for the 'Schrödinger operator'

$$
H=\Lambda i J \Lambda
$$

which is 'formally symmetric'.

2.3. Equivalence of reduction. In order to prove the equivalence of (1.2) and (2.10), we introduce the following new condition.

Condition III. $\quad J \mathcal{K} \subset \mathcal{V}$.

We denote by

$$
\Pi_{\mathcal{K}}: X \longrightarrow \mathcal{K}
$$

the orthogonal projection, and set

$$
P:=\Pi_{\mathcal{K}} J \Lambda .
$$


Lemma 2.1. Let conditions (1.4) and (2.12) hold. Then the operator $P: X \rightarrow \mathcal{V}$ is continuous.

Proof. It suffices to note that

$$
\Pi_{\mathcal{K}} J \Lambda=\sum_{1}^{N}\left|Y_{k}\right\rangle\left\langle Y_{k}\left|J \Lambda=-\sum_{1}^{N}\right| Y_{k}\right\rangle\left\langle\Lambda J Y_{k}\right|,
$$

where $Y_{k} \in \mathcal{K} \subset \mathcal{V}, N=\operatorname{dim} \mathcal{K}$, and $\Lambda J Y_{k} \in \mathcal{X}$ by (2.12).

Equation (2.7) with $X(t) \in C(\mathbb{R}, \mathcal{V})$ can be written as

$$
X(t)-X(0)=J \Lambda \int_{0}^{t} Z(s) d s, \quad t \in \mathbb{R} .
$$

By Lemma 2.1 this equation implies the system

$$
\left\{\begin{array}{l}
X_{\mathcal{R}}(t)-X_{\mathcal{R}}(0)=\left(1-\Pi_{\mathcal{K}}\right) J \Lambda \int_{0}^{t} Z(s) d s, \\
X_{\mathcal{K}}(t)-X_{\mathcal{K}}(0)=\Pi_{\mathcal{K}} J \Lambda \int_{0}^{t} Z(s) d s,
\end{array}\right.
$$

where

$$
X_{\mathcal{K}}(t)=\Pi_{\mathcal{K}} X(t) \quad \text { and } \quad X_{\mathcal{R}}(t)=\left(1-\Pi_{\mathcal{K}}\right) X(t) .
$$

Lemma 2.2. i) Let $X(t) \in C(\mathbb{R}, \mathcal{V})$ be a solution to (1.2) in the sense (2.7). Then $Z(t)=\Lambda X(t) \in C(\mathbb{R}, \mathcal{R})$ is the solution to (2.10) in the sense (2.9).

ii) Let $Z(t) \in C(\mathbb{R}, \mathcal{R})$ be a fixed solution to (2.10) in the sense (2.9). Then there exists a unique solution $X(t) \in C(\mathbb{R}, \mathcal{V})$ to (1.2) in the sense (2.7) satisfying (2.8).

Proof. It suffices to prove ii). The uniqueness holds, because

$$
X_{\mathcal{R}}(t)=\Lambda_{+}^{-1} Z(t), \quad X_{\mathcal{K}}(t)-X_{\mathcal{K}}(0)=\Pi_{\mathcal{K}} J \Lambda \int_{0}^{t} Z(s) d s,
$$

where the first equation follows from (2.8), and the second one, from the second equation of (2.15). 
To prove the existence we define $X_{\mathcal{R}}(t)$ and $X_{\mathcal{K}}(t)$ by (2.16). Then (2.8) holds, and $X(t)=X_{\mathcal{R}}(t)+X_{\mathcal{K}}(t) \in C(\mathbb{R}, \mathcal{V})$. Hence, the first equation (2.16) together with (2.9) and (2.8) imply that

$$
\begin{aligned}
X_{\mathcal{R}}(t)-X_{\mathcal{R}}(0) & =\Lambda_{+}^{-1}[Z(t)-Z(0)] \\
& =\Lambda_{+}^{-1} \Lambda J \Lambda \int_{0}^{t} Z(s) d s \\
& =\left(1-\Pi_{\mathcal{K}}\right) J \Lambda^{2} \int_{0}^{t} X(s) d s .
\end{aligned}
$$

Finally, the second equation (2.16) can be written as

$$
X_{\mathcal{K}}(t)-X_{\mathcal{K}}(0)=\Pi_{\mathcal{K}} J \Lambda^{2} \int_{0}^{t} X(s) d s
$$

by (2.8). Summing up, we obtain (2.7).

2.4. Symmetry and spectrum. The domain of $H$ is equal to

$$
D(H)=\{Z \in \mathcal{V}: J \Lambda Z \in \mathcal{V}\}=\Lambda_{\mathcal{R}}^{-1}(J \mathcal{V} \cap \mathcal{R})+\mathcal{K}
$$

Obviously, the operator $H$ is symmetric on $D(H)$, and hence, $H$ is a closable operator in $X$. However, we still do not know whether its domain is dense in $X$. This is why we need our last condition

Condition IV.

$$
H^{*}=H \text {. }
$$

A broad range of examples is provided by Lemma A.2. A concrete example is given by (4.11).

Theorem 2.3. Let conditions (1.3), (1.4), (2.12), and (2.21) hold. Then

$$
\sigma(H) \subset(-\infty,-\varepsilon] \cup 0 \cup[\varepsilon, \infty)
$$

with some $\varepsilon>0$. 
Proof. The operator

$$
\Lambda+\Pi_{\mathcal{K}}: \mathcal{V} \longrightarrow X
$$

is invertible by condition (1.3), since

$$
\left.\left(\Lambda+\Pi_{\mathcal{K}}\right)\right|_{\mathcal{K}}=\Pi_{\mathcal{K}} \quad \text { and }\left.\quad\left(\Lambda+\Pi_{\mathcal{K}}\right)\right|_{\mathcal{R}}=\Lambda_{+} .
$$

Hence, the operator

$$
H_{+}:=\left(\Lambda+\Pi_{\mathcal{K}}\right) i J\left(\Lambda+\Pi_{\mathcal{K}}\right)
$$

is also invertible; i.e., its inverse

$$
H_{+}^{-1}:=\left(\Lambda+\Pi_{\mathcal{K}}\right)^{-1} i J\left(\Lambda+\Pi_{\mathcal{K}}\right)^{-1}
$$

is a bounded operator on $X$. On the other hand, this operator is symmetric on $X$, and hence it is selfadjoint. Moreover, $H_{+}$is injective operator on $X$. Hence, Theorem 13.11 (b) of [37] implies that $H_{+}$is a selfadjoint operator with a dense domain $D\left(H_{+}\right)$. Further,

$$
\begin{aligned}
H_{+} & =H+\Pi_{\mathcal{K}} i J\left(\Lambda+\Pi_{\mathcal{K}}\right)+\left(\Lambda+\Pi_{\mathcal{K}}\right) i J \Pi_{\mathcal{K}}+\Pi_{\mathcal{K}} i J \Pi_{\mathcal{K}} \\
& =H+T .
\end{aligned}
$$

Here, $\Pi_{\mathcal{K}} i J\left(\Lambda+\Pi_{\mathcal{K}}\right)$ and $\Pi_{\mathcal{K}} i J \Pi_{\mathcal{K}}$ are finite-rank operators $\mathcal{V} \rightarrow \mathcal{V}$. On the other hand, (2.12) implies that $\left(\Lambda+\Pi_{\mathcal{K}}\right) i J \Pi_{\mathcal{K}}$ is also a finite-rank operator from $\mathcal{V}$ to $\mathcal{V}$. Hence, $T: \mathcal{V} \rightarrow \mathcal{V}$ is the finite-rank operator which is symmetric in $X$. As the result, (2.25) implies that $H$ is defined and symmetric on $D\left(H_{+}\right)$.

Further, the resolvent

$$
\left(H_{+}-\lambda\right)^{-1}: X \longrightarrow X
$$

is bounded and analytic in a small complex neighborhood $\mathcal{O}$ of $\lambda=0$, and

$$
H-\lambda=H_{+}-\lambda-T=\left[1-T\left(H_{+}-\lambda\right)^{-1}\right]\left(H_{+}-\lambda\right), \quad \lambda \in \mathcal{O} .
$$

Here, the operator $H-\lambda$ is invertible for $\operatorname{Im} \lambda \neq 0$ by (2.21), while $H_{+}-\lambda$ is invertible in a small complex neighborhood $\mathcal{O}$ of $\lambda=0$. Hence,

$$
\operatorname{Ker}\left[1-T\left(H_{+}-\lambda\right)^{-1}\right]=0 \quad \text { for } \lambda \in \mathcal{O},
$$

with $\operatorname{Im} \lambda \neq 0$. Therefore, $1-T\left(H_{+}-\lambda\right)^{-1}$ is invertible for these $\lambda$ by Fredholm's theorem, inasmuch as $T$ is a finite-rank operator. Hence, it is also invertible in $\mathcal{O}$ outside a discrete set. Now (2.26) implies (2.22). 
Remark 2.4. Let conditions (1.3), (1.4), and (2.12) hold.

(1) The domain of $H$ is dense in $X$, as is shown in the proof of Theorem 2.3.

(2) $H$ admits selfadjoint extensions, because

$$
N_{+}=N_{-}, \quad N_{ \pm}:=\operatorname{dim}[\operatorname{Ran}(H \mp i)]^{\perp} .
$$

Indeed, $\operatorname{Ran}\left(H_{+}-\lambda\right)=\chi$ for $\lambda$ from a small complex neighborhood $\mathcal{O}$ of $\lambda=0$. On the other hand, the dimension of $\left(\operatorname{Ran}\left[1-T\left(H_{+}-\lambda\right)^{-1}\right]\right)^{\perp}$ is constant in $\mathcal{O}$ outside a discrete set, because $T$ is a finite-rank operator. Therefore, (2.26) implies that $\operatorname{dim}[\operatorname{Ran}(H-\lambda)]^{\perp}$ is also constant in $\mathcal{O}$ outside a discrete set, verifying (2.27).

\section{Dynamical group and canonical form}

We construct spectral representation for solutions to (1.2) and deduce the canonical form of the Hamilton generator.

3.1. Spectral representation of solutions. We will construct solutions to (2.10), and afterwords, reconstruct the corresponding solutions to (1.2). The Spectral Theorem implies the following lemma.

Lemma 3.1. Let conditions (1.3), (1.4), (2.12), and (3.1) hold. Then, for any $Z(0) \in$ $\mathcal{R}$, equation (2.10) admits a unique solution $Z(t) \in C(\mathbb{R}, \mathcal{R})$ in the sense (2.9). The solution is given by

$$
Z(t)=e^{-i H t} Z(0) \in C(\mathbb{R}, \mathcal{R}) .
$$

Now we can reconstruct solutions to (1.2) using formulas (2.16):

$$
X(t)=\Lambda_{+}^{-1} e^{-i H t} \Lambda X(0)+X_{\mathcal{K}}(0)+P \int_{0}^{t} e^{-i H s} \Lambda X(0) d s
$$

where the operator $P: \mathcal{R} \rightarrow \mathcal{V}$ is bounded by Lemma 2.1. To evaluate the integral in (3.2), we denote by $\Pi_{0}$ and $\Pi_{R}$, respectively, the spectral projections of $\mathcal{X}$ onto Ker $H \cap \mathcal{R}$ and onto $R:=\operatorname{Ran} H \subset \mathcal{R}$. Obviously,

$$
e^{-i H s}=\Pi_{0}+e^{-i H_{R} s} \Pi_{R}, \quad \int_{0}^{t} e^{-i H s} d s=t \Pi_{0}+i\left(e^{-i H_{R} t}-1\right) H_{R}^{-1} \Pi_{R},
$$


where

$$
H_{R}:=\left.H\right|_{R \cap D(H)}
$$

Now (3.2) reads

$$
\begin{aligned}
X(t)= & \Lambda_{+}^{-1} e^{-i H t} \Lambda X(0)+X_{\mathcal{K}}(0) \\
& +t P \Pi_{0} \Lambda X(0)+i P\left(e^{-i H_{R} t}-1\right) H_{R}^{-1} \Pi_{R} \Lambda X(0) .
\end{aligned}
$$

Lemmas 3.1 and 2.2 imply the following proposition.

Proposition 3.2. Let conditions (1.3), (1.4), (2.12), and (3.1) hold. Then, for any $X(0) \in \mathcal{V}$,

i) equation (1.2) admits a unique solution $X(t) \in C(\mathbb{R}, \mathcal{V})$ and

ii) the solution admits the spectral representation (3.4).

3.2. Spectral resolution. Representation (3.4) can be written as

$$
\begin{aligned}
e^{A t}=\Lambda_{+}^{-1} \int_{\mathbb{R}} e^{-i \omega t} d E(\omega) \Lambda+\Pi_{\mathcal{K}} \\
\quad+t P \Pi_{0} \Lambda+i P \int_{|\omega| \geq \varepsilon} \frac{e^{-i \omega t}-1}{\omega} d E(\omega) \Lambda,
\end{aligned}
$$

where $d E(\omega)$ denotes the spectral family of $H$, and $\varepsilon>0$ is the number from equation (2.22). Formally,

$$
e^{A t}=\int_{\mathbb{R}} e^{-i \omega t} d F(\omega),
$$

where

$$
\begin{aligned}
d F(\omega)=\left[\Lambda_{+}^{-1}+\frac{i P}{\omega}\right] \chi_{\varepsilon}(\omega) d E(\omega) \Lambda \\
\quad+\left[\Pi_{\mathcal{K}}-i P \int_{|\omega| \geq \varepsilon} \frac{d E(\omega)}{\omega} \Lambda\right] \delta(\omega) d \omega \\
\quad+\Lambda_{+}^{-1} \Pi_{0} \Lambda \delta(\omega) d \omega-i P \Pi_{0} \Lambda \delta^{\prime}(\omega) d \omega,
\end{aligned}
$$

and $\chi_{\varepsilon}$ is the indicator of the set $|\omega| \geq \varepsilon$. Setting $t=0$ in both sides of (3.6) and in their derivatives, we formally obtain

$$
1=\int_{\mathbb{R}} d F(\omega), \quad A=-i \int_{\mathbb{R}} \omega d F(\omega) .
$$


3.3. Canonical form. First we will identify the eigenvectors and the associated eigenvectors of A formally relying on (3.8). Afterwords, we will prove the identifications rigorously.

The set

$$
\mathcal{W}:=\{X \in \mathcal{V}: \Lambda X \in D(H)\}
$$

is dense in $V$ under our conditions (1.3) and (1.4). Let us apply the both sides of identities (3.8) to an arbitrary $X \in \mathcal{W}$. Using (3.7), we formally obtain

$$
X=\int_{\mathbb{R}} d F(\omega) X=X_{\varepsilon}+X_{0}+X_{a}
$$

and

$$
A X=-i \int_{\mathbb{R}} \omega d F(\omega) X=A X_{\varepsilon}+A X_{0}+A X_{a}
$$

where

$$
\begin{array}{rlr}
X_{\varepsilon} & :=\int_{|\omega| \geq \varepsilon}\left[\Lambda_{+}^{-1}+\frac{i P}{\omega}\right] d E(\omega) \Lambda X \\
A X_{\varepsilon} & =-i \int_{|\omega| \geq \varepsilon}\left[\omega \Lambda_{+}^{-1}+i P\right] d E(\omega) \Lambda X \\
X_{0} & :=\left[\Pi_{\mathcal{K}}-i P \int_{|\omega| \geq \varepsilon} \frac{d E(\omega)}{\omega} \Lambda\right] X, \quad A X_{0}=0, \\
X_{a} & :=\Lambda_{+}^{-1} \Pi_{0} \Lambda X, & A X_{a}=P \Pi_{0} \Lambda X .
\end{array}
$$

Here, (3.10) means the expansion over the eigenvectors with eigenvalues $-i \omega$, while (3.11), with the zero eigenvalue. Formula (3.12) means that $X_{a}$ is the associated eigenvector to the eigenvector $P \Pi_{0} \Lambda X$, which corresponds to the zero eigenvalue. We justify the formal calculations (3.9)-(3.12) in the following lemma.

Lemma 3.3. Formulas (3.9)-(3.12) hold for $X \in \mathcal{W}$.

Proof. i) Formulas (3.9) and (3.11) are obvious.

ii) The last formula of (3.12) follows from the fact that $A X_{a}=J \Lambda \Pi_{0} \Lambda X=$ $\Pi_{\mathcal{K}} J \Lambda \Pi_{0} \Lambda X$, since $\Lambda J \Lambda \Pi_{0} \Lambda X=-i H \Pi_{0} \Lambda X=0$ by definition of $\Pi_{0}$.

iii) Finally, let us prove (3.10). The representation (3.4) implies that $\dot{X}(\cdot) \in$ $C(\mathbb{R}, \mathcal{V})$ for $X(0) \in \mathcal{W}$ since

$$
\dot{X}(t)=-i \Lambda_{+}^{-1} e^{-i H t} H \Lambda X(0)+P \Pi_{0} \Lambda X(0)+P e^{-i H_{R} t} \Pi_{R} \Lambda X(0)
$$


by Hille-Yosida's theorem [37, Theorem 13.35 (c)]. On the other hand, according to $(2.7)$,

$$
\frac{X(t+\Delta t)-X(t)}{\Delta t}=A \frac{\int_{t}^{t+\Delta t} X(s) d s}{\Delta t} .
$$

Here the left hand side converges to $\dot{X}(t)$ in $\mathcal{V}$ as $\Delta t \rightarrow 0$, since $\dot{X}(\cdot) \in C(\mathbb{R}, \mathcal{V})$, and the quotient on the right converges to $X(t)$ in $\mathcal{V}$ by (2.6). Hence, making $\Delta t \rightarrow 0$ we obtain

$$
\dot{X}(t)=A X(t),
$$

since the operator $A=J B$ is closed in $X$. Setting $t=0$ in (3.13) and (3.15), and writing $X$ instead of $X(0)$, we obtain

$$
\begin{aligned}
A X & =-i \Lambda_{+}^{-1} H \Lambda X+P \Pi_{0} \Lambda X+P \Pi_{R} \Lambda X \\
& =-i \int_{|\omega| \geq \varepsilon}\left[\omega \Lambda_{+}^{-1}+i P\right] d E(\omega) \Lambda X+P \Pi_{0} \Lambda X, \quad X \in \mathcal{W} .
\end{aligned}
$$

On the other hand, $A X=A X_{\varepsilon}+A X_{0}+A X_{a}=A X_{\varepsilon}+P \Pi_{0} \Lambda X$ by (3.11) and (3.12). Hence, (3.16) implies (3.10).

Corollary 3.4. The nontrivial Jordan blocks occur only for $\lambda=0$; they are of size $2 \times 2$ (in accordance with [29, Proposition 5.1]), and their number is

$$
\operatorname{dim} \operatorname{Ker} H_{R}=\operatorname{dim}[\operatorname{Ker} H \cap \mathcal{R}],
$$

where

$$
H_{R}:=\left.H\right|_{R \cap D(H)} .
$$

This number is finite by (1.4).

Further, we set $\Pi_{\mathcal{R}}:=1-\Pi_{\mathcal{K}}$ and introduce the 'Green operator'

$$
G:=\Lambda_{+}^{-1} \Pi_{\mathcal{R}}+i P H_{R}^{-1} \Pi_{R} .
$$

It is continuous from $X$ to $\mathcal{V}$ by our conditions (1.3) and (2.12) and by Lemma 2.1. Therefore, formulas (3.10) can be rewritten as

$$
X_{\varepsilon}=G \int_{|\omega| \geq \varepsilon} d E(\omega) \Lambda X, \quad A X_{\varepsilon}=-i G \int_{|\omega| \geq \varepsilon} \omega d E(\omega) \Lambda X, \quad X \in \mathcal{W},
$$

since both these integrals converge in $X$ and belong to $R \subset \mathcal{R}$. 
Corollary 3.5. Let $h_{k} \in X$ be an eigenfunction of $H$ corresponding to an eigenvalue $\omega_{k} \neq 0$. Then

$$
a_{k}:=G h_{k} \in \mathcal{V}
$$

is the eigenfunction of $A$ corresponding to the eigenvalue $-i \omega_{k}$.

\section{Application to eigenfunction expansion}

We are going to apply our results to justify the eigenfunction expansion (1.6) in the context of the system considered in [22]. We have used this expansion for the calculation of the Fermi Golden Rule [22, (5.14)].

4.1. Linearization at the kink. In $[22,23]$ we studied the $1 \mathrm{D}$ relativistic Ginzburg-Landau equation

$$
\ddot{\psi}(x, t)=\frac{d^{2}}{d x^{2}} \psi(x, t)+F(\psi(x, t)), \quad x \in \mathbb{R}
$$

for real solutions $\psi(x, t)$. Here, $F(\psi)=-U^{\prime}(\psi)$, where $U(\psi)$ is similar to the Ginzburg-Landau potential $U_{G L}(\psi)=\left(\psi^{2}-1\right)^{2} / 4$, which corresponds to the cubic equation with $F(\psi)=\psi-\psi^{3}$. Namely, $U(\psi)$ is a real smooth even function satisfying the following conditions:

$$
\begin{cases}U(\psi)>0, & \psi \neq \pm a, \\ U(\psi)=\frac{m^{2}}{2}(\psi \mp a)^{2}+O\left(|\psi \mp a|^{14}\right), & x \rightarrow \pm a,\end{cases}
$$

where $a, m>0$. The main goal of $[22,23]$ was to prove the asymptotic stability of solitons (kinks) $\psi(x, t)=s_{v}(x-v t)$ that move with constant velocity $|v|<1$, and

$$
s_{v}(x) \longrightarrow \pm a, \quad x \rightarrow \pm \infty .
$$

Substituting $\psi(x, t)=s_{v}(x-v t)$ into (4.1), we obtain the corresponding stationary equation

$$
v^{2} s_{v}^{\prime \prime}(x)=s_{v}^{\prime \prime}(x)+F\left(s_{v}(x)\right), \quad x \in \mathbb{R} .
$$

The linearization of (4.1) at the kink $s_{v}(x-v t)$ in the moving frame reads as (1.2) with $X=(\psi, \dot{\psi}) \in L^{2}(\mathbb{R}) \otimes \mathbb{C}^{2}$ (for the corresponding complexification) and with the generator [23, (4.6)]

$$
A_{v}=\left(\begin{array}{cc}
v \frac{d}{d x} & 1 \\
\frac{d^{2}}{d x^{2}}-m^{2}-V_{v}(x) & v \frac{d}{d x}
\end{array}\right) .
$$


Here, the potential

$$
V_{v}(x)=-F^{\prime}\left(s_{v}(x)\right)-m^{2} \in C^{\infty}(\mathbb{R}) .
$$

The kink $s_{v}(x)$ is an odd monotone function in a suitable coordinate $x$, while $F^{\prime}(\psi)=-U^{\prime \prime}(\psi)$ is an even function of $\psi$. Hence, the potential $V_{v}(x)$ is an even function of $x$. Moreover,

$$
\left|V_{v}(x)\right| \leq C e^{-\kappa|x|}, \quad x \in \mathbb{R},
$$

where $\kappa>0$. The generator (4.5) has the form $A_{v}=J B_{v}$ with

$$
B_{v}=\left(\begin{array}{cc}
S_{v} & -v \frac{d}{d x} \\
v \frac{d}{d x} & 1
\end{array}\right), \quad J:=\left(\begin{array}{cc}
0 & 1 \\
-1 & 0
\end{array}\right),
$$

where

$$
S_{v}:=-\frac{d^{2}}{d x^{2}}+m^{2}+V_{v}(x) .
$$

Obviously, $J B_{v} \neq B_{v} J$. Differentiating (4.4), we obtain

$$
\left[S_{v}+v^{2} \frac{d^{2}}{d x^{2}}\right] s_{v}^{\prime}(x)=0
$$

4.2. Spectral conditions. Conditions (1.4) and (2.12) hold for operators (4.8) on $X:=L^{2}(\mathbb{R}) \otimes \mathbb{C}^{2}$ by Lemma A.2. Condition (1.3) for all $|v|<1$ follows from Lemmas A.1 and A.2 of [26]. Here, we check (1.3) in the case $v=0$ for the completeness of the exposition. We will write $A, B$ and $S$, respectively, instead of $A_{0}, B_{0}$ and $S_{0}$ :

$$
A=\left(\begin{array}{cc}
0 & 1 \\
-S & 0
\end{array}\right), \quad B=\left(\begin{array}{cc}
S & 0 \\
0 & 1
\end{array}\right)
$$

where

$$
S:=-\frac{d^{2}}{d x^{2}}+m^{2}+V_{0}(x)
$$

The operators $B$ and $S$ are essentially selfadjoint in $L^{2}(\mathbb{R}) \otimes \mathbb{C}^{2}$ and $L^{2}(\mathbb{R})$, respectively, by (4.7) and Theorems X.7 and X.8 of [35]. We will consider the closures of $B$ and $S$, which are both selfadjoint. In this case,

$$
\Lambda:=B^{1 / 2}=\left(\begin{array}{cc}
\sqrt{S} & 0 \\
0 & 1
\end{array}\right), \quad H:=\Lambda i J \Lambda=i\left(\begin{array}{cc}
0 & \sqrt{S} \\
-\sqrt{S} & 0
\end{array}\right)=i J \sqrt{S} .
$$

Hence, the operator $H$ is also selfadjoint on the domain $D(\sqrt{S}) \oplus D(\sqrt{S})$. Thus, condition (2.21) holds in our case. 
Lemma 4.1. Condition (1.3) holds for the operator $B$ on $X=L^{2}(\mathbb{R}) \otimes \mathbb{C}^{2}$.

Proof. Equation (4.9) with $v=0$ means that $\lambda=0 \in \sigma_{p p}(S)$. Moreover, $\lambda=0$ is the minimal eigenvalue of $S$, since the corresponding eigenfunction $s_{0}^{\prime}(x)$ does not vanish [23, (1.9)]. Hence,

$$
\sigma(S) \subset[0, \infty), \quad \operatorname{Ker} S=\left(s_{0}^{\prime}(x)\right) .
$$

Further, the continuous spectrum of $S$ lies in $\left[\mathrm{m}^{2}, \infty\right)$, and hence (4.12) implies

$$
\sigma(S)=\left\{\lambda_{0}, \ldots, \lambda_{N}\right\} \cup\left[m^{2}, \infty\right),
$$

where $0=\lambda_{0}<\cdots<\lambda_{N}<m^{2}$. Finally, $\sigma(B)=\sigma(S) \cup\{1\}$, by (1.3).

We will assume below the following spectral condition (imposed in [22])) at the edge point of the continuous spectrum of $S$ :

the point $m^{2}$ is neither an eigenvalue nor a resonance of $S$.

This condition provides a regularity of the eigenvalue expansion (1.6) at the edge points $\pm m$ of the continuous spectrum.

\section{Orthogonal eigenfunction expansion}

We are going to apply Proposition 3.2 to the case of operators (4.10). First, (4.13) implies that

$$
\sigma(H)=(-\infty,-m] \cup\left\{\omega_{-N}, \ldots, \omega_{-1}, \omega_{0}, \omega_{1}, \ldots, \omega_{N}\right\} \cup[m, \infty),
$$

where $\omega_{ \pm k}^{2}=\lambda_{k}$ for $k=0, \ldots, N$. We denote by

$$
\sigma_{c}=(-\infty,-m] \cup[m, \infty)
$$

the continuous spectrum of $H$, and

$$
\Psi_{0}=\Lambda_{+}^{-1} \Pi_{0} \Lambda X(0), \quad \Phi_{0}=P \Pi_{0} \Lambda X(0) .
$$

Then $A \Psi_{0}=0$ and $A \Psi_{0}=\Phi_{0}$ by (3.12), and hence, $t \Phi_{0}+\Psi_{0}$ is the solution to (1.2). Now formula (3.5) can be rewritten as

$$
\begin{aligned}
X(t)= & e^{A t} X(0) \\
= & t \Phi_{0}+\Psi_{0}+\sum_{-N}^{N} e^{-i \omega_{k} t} C_{k} a_{k} \\
& +\int_{\sigma_{c}}\left[\Lambda_{\mathcal{R}}^{-1}+\frac{i P}{\omega}\right] e^{-i \omega t} d E(\omega) \Lambda X(0) .
\end{aligned}
$$


Here, $a_{0} \in \mathcal{K}$ and

$$
a_{k}=\left[\Lambda_{\mathcal{R}}^{-1}+\frac{i P}{\omega_{k}}\right] h_{k}=G h_{k} \in \mathcal{X}, \quad k \neq 0,
$$

where $h_{k} \in \mathcal{R}$ are the eigenfunctions of $H$ corresponding to the eigenvalues $\omega_{k} \neq 0$. By Corollary 3.5, $a_{k}$ are the eigenfunctions of $A$ corresponding to the eigenvalues $-i \omega_{k}$.

Let us denote by $X^{c}(t)$ the integral in (5.3):

$$
X^{c}(t)=\int_{\sigma_{c}}\left[\Lambda_{\mathcal{R}}^{-1}+\frac{i P}{\omega}\right] e^{-i \omega t} d E(\omega) \Lambda X(0) .
$$

To prove (1.6), it remains to justify the eigenfunction expansion

$$
X^{c}(t)=\int_{\sigma_{c}} e^{-i \omega t} C(\omega) a_{\omega} d \omega
$$

where $a_{\omega}$ are the generalized eigenfunctions of $A$ corresponding to the eigenvalues $-i \omega$, and the meaning of the convergence of the integral will be specified later. Then (1.6) will follow from (5.3).

By (5.3), the function $X^{c}(t)$ is the solution to (1.2), and hence

$$
Z^{c}(t):=\Lambda X^{c}(t)=\int_{\sigma_{c}} e^{-i \omega t} d E(\omega) \Lambda X(0)
$$

is the solution to (2.10). We will deduce (5.6) from the corresponding representation

$$
Z^{c}(t)=\int_{\sigma_{c}} e^{-i \omega t} C(\omega) h_{\omega} d \omega
$$

where $h_{\omega}$ are generalized eigenfunctions of $H$ corresponding to the eigenvalues $\omega$ normalized by

$$
\left\langle h_{\omega}, h_{\omega^{\prime}}\right\rangle=2 \pi \delta\left(\omega-\omega^{\prime}\right), \quad \omega, \omega^{\prime} \in \sigma_{c} .
$$

The normalization means by definition, that

$$
\left\langle Z_{1}, Z_{2}\right\rangle=2 \pi \int_{m \leq|\omega| \leq M} C_{1}(\omega) \overline{C_{2}(\omega)} d \omega \quad \text { for } Z_{k}=\int_{m \leq|\omega| \leq M} C_{k}(\omega) h_{\omega} d \omega \in \mathcal{X}
$$


For $\rho \in \mathbb{R}$ we denote by

$$
L_{\rho}^{2}=L_{\rho}^{2}(\mathbb{R})
$$

the weighted Hilbert space with the norm

$$
\|\psi\|_{L_{\rho}^{2}}^{2}:=\int\langle x\rangle^{2 \rho}|\psi(x)|^{2} d x, \quad\langle x\rangle:=\left(1+x^{2}\right)^{1 / 2} .
$$

Theorem 5.1. Let condition (4.14) hold and $s>1$. Then, for $\omega \in \sigma_{c}$, there exists $h_{\omega} \in L_{-s}^{2} \otimes \mathbb{C}^{2}$ satisfying the following conditions.

i) $h_{\omega}$ is a continuous function of $\omega \in \sigma_{c}$ with values in $L_{-s}^{2} \otimes \mathbb{C}^{2}$.

ii) The normalization (5.9) holds.

iii) $h_{\omega}$ are the generalized eigenfunctions of $H$, i.e.,

$$
H Z=\int_{\sigma_{c}} \omega C(\omega) h_{\omega} d \omega \quad \text { if } Z=\int_{\sigma_{c}} C(\omega) h_{\omega} d \omega \in D(H) .
$$

iv) The eigenfunction expansion (5.8) holds in the following sense:

$$
\left\|Z^{c}(t)-\int_{m \leq|\omega| \leq M} e^{-i \omega t} C(\omega) h_{\omega} d \omega\right\|_{L^{2} \otimes \mathbb{C}^{2}} \longrightarrow 0, \quad M \rightarrow \infty .
$$

Proof. i) We construct the generalized eigenfunctions and the eigenfunction expansion (5.8) by solving equation (2.10) for $Z^{c}(t)=\left(Z_{1}^{c}(t), Z_{2}^{c}(t)\right)$. By (4.11), the equation is equivalent to the system

$$
\dot{Z}_{1}^{c}(t)=\sqrt{S} Z_{2}^{c}(t), \quad \dot{Z}_{2}^{c}(t)=-\sqrt{S} Z_{1}^{c}(t) .
$$

Eliminating $Z_{2}^{c}(t)$, we obtain

$$
\ddot{Z}_{1}^{c}(t)=-S Z_{1}^{c}(t)
$$

Further we apply Theorem XI.41 of [36] and the arguments of [36, pp 114-115]. Namely, the rapid decay (4.7) and our spectral condition (4.14) imply the following Limiting Absorption Principle (LAP) [1, 24, 36]:

$$
R(\lambda \pm i \varepsilon) \longrightarrow R_{ \pm}(\lambda), \quad \varepsilon \rightarrow+0, \lambda \in\left[m^{2}, \infty\right),
$$

where

$$
R(z):=(S-z)^{-1}
$$


and the convergence holds in the strong topology of the space of continuous operators $L_{s}^{2} \rightarrow L_{-s}^{2}$ with $s>1$. Moreover, the traces of the resolvent $R_{ \pm}(\lambda)$ are continuous functions of $\lambda \geq m^{2}$ with values in $L\left(L_{s}^{2}, L_{-s}^{2}\right)$. The continuity at $\lambda>0$ has been established by Agmon, see $[1,24]$. The continuity at $\lambda=0$ under condition (4.14) is proved in [25, formulas (3.12)]. The LAP serves as the basis for the eigenfunction expansion

$$
\begin{aligned}
Z_{1}^{c}(t) & =\int_{\sigma_{c}} d \mathcal{E}\left(\omega^{2}\right)\left[Z_{1}^{c}(0) \cos \omega t+Z_{2}^{c}(0) \sin \omega t\right] \\
& =\int_{\sigma_{c}} e^{-i \omega t} C(\omega) e_{\omega} d \omega
\end{aligned}
$$

where $d \mathcal{E}(\lambda)$ is the spectral resolution of $S$, while $e_{\omega} \in L_{-s}^{2}$ are generalized eigenfunctions of $S$ corresponding to the eigenvalues $\omega^{2} \geq m^{2}$. Here the first identity follows by Spectral Theorem, while the second follows by Theorem XI.41 (e) of [36]. The eigenfunctions are defined by formulas of [36, pp 114-115]:

$$
e_{\omega}=W^{*}(\omega) f_{\omega}, \quad W(\omega):=\left[1+V R_{0}\left(\omega^{2}+i 0\right)\right]^{-1}, \omega \in \sigma_{c} .
$$

where

$$
f_{\omega}(x):=\sin |\omega| x \quad \text { and } \quad R_{0}(\lambda):=\left(-\Delta+m^{2}-\lambda\right)^{-1} .
$$

The operator $W(\omega)$ is a continuous function of $\omega \in \sigma_{c}$ with values in $L\left(L_{s}^{2}, L_{s}^{2}\right)$ by the formula

$$
\left[1+V R_{0}(\lambda)\right]^{-1}=1-V R(\lambda)
$$

and the decay (4.7). Respectively, the adjoint operator $W^{*}(\omega)$ is a continuous function of $\omega \in \sigma_{c}$ with values in $L\left(L_{-s}^{2}, L_{-s}^{2}\right)$. As the result, $e_{\omega}$ is a continuous function of $\omega \in \sigma_{c}$ with values in $L_{-s}^{2}$. The normalization of $e_{\omega}$ coincides with the same of the 'free' generalized eigenfunctions $f_{\omega}$ :

$$
\left\langle e_{\omega}, e_{\omega^{\prime}}\right\rangle=\pi \delta\left(|\omega|-\left|\omega^{\prime}\right|\right), \quad \omega, \omega^{\prime} \in \sigma_{c},
$$

which follows from the last formula on page 115 of [36]. Finally, Theorem XI.41 (e) of [36] implies that the last integral (5.17) converges in $L^{2}=L^{2}(\mathbb{R})$ :

$$
\left\|Z_{1}^{c}(t)-\int_{m \leq|\omega| \leq M} e^{-i \omega t} C(\omega) e_{\omega} d \omega\right\|_{L^{2}} \longrightarrow 0, \quad M \rightarrow \infty .
$$

Now (5.8) for $Z_{1}^{c}(t)$ follows from (5.17). For $Z_{2}^{c}(t)$ we use the first equation of (5.14), which implies

$$
Z_{2}^{c}(t)=-i \int_{\sigma_{c}} \operatorname{sgn} \omega e^{-i \omega t} C(\omega) e_{\omega} d \omega .
$$


Combining (5.17) and (5.22), we obtain (5.8) with

$$
h_{\omega}:=\left(\begin{array}{c}
1 \\
-i \operatorname{sgn} \omega
\end{array}\right) e_{\omega},
$$

which is the continuous function of $\omega \in \sigma_{c}$ with values in $L_{-s}^{2} \otimes \mathbb{C}^{2}$.

ii) Normalization (5.9) follows from (5.20).

iii) $Z^{c}(t) \in D(H)$ means that $Z_{1,2}^{c}(t) \in D(\sqrt{S})$. Furthermore,

$$
H Z^{c}(t)=i \sqrt{S}\left(\begin{array}{c}
Z_{2}^{c}(t) \\
-Z_{1}^{c}(t)
\end{array}\right) \text {. }
$$

Now (5.12) follows from the expansions (5.17) and (5.22) for $Z_{1,2}^{c}(t)$ by [36, Theorem XI.41 (c)], since $e_{\omega}$ are the generalized eigenfunctions of $S$ with the eigenvalues $\omega^{2}$, and formally,

$$
i \sqrt{S}\left(\begin{array}{c}
-i \operatorname{sgn} \omega \\
-1
\end{array}\right) e_{\omega}=\left(\begin{array}{c}
\operatorname{sgn} \omega \\
-i
\end{array}\right)|\omega| e_{\omega}=\omega h_{\omega} .
$$

iv) (5.13) follows from (5.21) and similar convergence for $Z_{2}^{c}$.

\section{Nonorthogonal eigenfunction expansion}

Let us denote by $Z_{M}^{c}(t, x)$ the integral in (5.13). This integral is defined for almost all $x$; i.e.,

$$
Z_{M}^{c}(t, x):=\int_{m \leq|\omega| \leq M} e^{-i \omega t} C(\omega) h_{\omega}(x) d \omega, \quad \text { a.a. } x \in \mathbb{R} .
$$

To justify (5.6) we should adjust the meaning of this integral relying on the following lemma, which is proved in [26].

Lemma 6.1 ([26, Lemma 5.1]). Let condition (4.14) hold. Then

i) The integral (6.1) converges absolutely in $L_{-s}^{2} \otimes \mathbb{C}^{2}$ for every $s>1$ :

$$
\int_{m \leq|\omega| \leq M}\left\|C(\omega) h_{\omega}\right\|_{L_{-s}^{2} \otimes \mathbb{C}^{2}} d \omega<\infty, \quad M>m ;
$$

ii) the integral of these $L_{-s}^{2} \otimes \mathbb{C}^{2}$-valued functions over $m \leq|\omega| \leq M$ coincides with (6.1) almost everywhere.

Further, we express $X^{c}(t)$ in terms of $Z^{c}(t)$ and the Green operator (3.17), and prove the appropriate continuity of $G$, which allows us to deduce (5.6) from (5.13). 
6.1. Reconstruction via the Green operator. Similarly to (3.18), we use (5.7) to rewrite integral (5.5) as

$$
X^{c}(t)=G \int_{\sigma_{c}} e^{-i \omega t} d E(\omega) \Lambda X(0)=G Z^{c}(t),
$$

taking into account that $Z^{c}(t) \in R \subset \mathcal{R}$ and that the Green operator $G: X \rightarrow \mathcal{V}$ is continuous. Now (5.8) implies that

$$
X^{c}(t)=G \int_{\sigma_{c}} e^{-i \omega t} C(\omega) h_{\omega} d \omega .
$$

Similarly to (3.18),

$$
\begin{aligned}
A X^{c}(t) & =-i G \int_{\sigma_{c}} e^{-i \omega t} \omega d E(\omega) \Lambda X(0) \\
& =-i G H \int_{\sigma_{c}} e^{-i \omega t} d E(\omega) \Lambda X(0)=-i G H Z^{c}(t), \quad X(0) \in \mathcal{W} .
\end{aligned}
$$

Therefore, (5.12) gives

$$
A X^{c}(t)=-i G \int_{\sigma_{c}} e^{-i \omega t} \omega C(\omega) h_{\omega} d \omega .
$$

6.2. Continuity of the Green operator. Now we are going to establish the continuity of the Green operator $G$ in the weighted norms (5.11). We will simplify the form of $G$ in the concrete case (4.11) by proving that

$$
P H_{R}^{-1}=\Pi_{\mathcal{K}} J \Lambda H_{R}^{-1}=0 .
$$

First we note that

$$
\operatorname{Ker} H=\operatorname{Ker} S \oplus \operatorname{Ker} S, \quad R=\operatorname{Ran} H=\operatorname{Ran} S \oplus \operatorname{Ran} S
$$

by (4.11). Further, we set

$$
S_{+}:=\left.S\right|_{\operatorname{Ran} S}: \operatorname{Ran} S \cap D(S) \rightarrow \operatorname{Ran} S,
$$

and let $P_{0}$ denote the orthogonal projection of $L^{2}(\mathbb{R})$ onto $\operatorname{Ker} S$. Then

$$
P_{+}:=1-P_{0}
$$


is the orthogonal projection of $L^{2}(\mathbb{R})$ onto $\operatorname{Ran} S$, and now (4.11) implies

$$
\begin{aligned}
\Pi_{\mathcal{K}}=\left(\begin{array}{cc}
P_{0} & 0 \\
0 & 0
\end{array}\right), & \Pi_{\mathcal{R}}=\left(\begin{array}{cc}
P_{+} & 0 \\
0 & 1
\end{array}\right), \\
\Pi_{0}=\left(\begin{array}{cc}
P_{0} & 0 \\
0 & P_{0}
\end{array}\right), & \Pi_{R}=\left(\begin{array}{cc}
P_{+} & 0 \\
0 & P_{+}
\end{array}\right) .
\end{aligned}
$$

Hence, finally,

$$
H_{R}^{-1}=i\left(\begin{array}{cc}
0 & S_{+}^{-1 / 2} \\
-S_{+}^{-1 / 2} & 0
\end{array}\right)
$$

by (4.11), and therefore,

$$
J \Lambda H_{R}^{-1}=i\left(\begin{array}{cc}
0 & 1 \\
-1 & 0
\end{array}\right)\left(\begin{array}{cc}
S_{+}^{1 / 2} & 0 \\
0 & 1
\end{array}\right)\left(\begin{array}{cc}
0 & S_{+}^{-1 / 2} \\
-S_{+}^{-1 / 2} & 0
\end{array}\right)=-i\left(\begin{array}{cc}
S_{+}^{-1 / 2} & 0 \\
0 & P_{+}
\end{array}\right) .
$$

Applying $\Pi_{\mathcal{K}}$, we get (6.7).

Now definition (3.17) implies that

$$
G=\Lambda_{+}^{-1} \Pi_{\mathcal{R}}
$$

The following lemma is a generalization of [26, Lemma 5.2].

Lemma 6.2. The operator

$$
G: L_{\rho}^{2} \otimes \mathbb{C}^{2} \longrightarrow L_{\rho}^{2} \otimes \mathbb{C}^{2}
$$

is continuous for every $\rho \in \mathbb{R}$.

Proof. Using the first formula of (4.11) and the formula for $\Pi_{\mathcal{R}}$, we get

$$
\Lambda_{\mathcal{R}}^{-1} \Pi_{\mathcal{R}}=\left(\begin{array}{cc}
S_{+}^{-1 / 2} P_{+} & 0 \\
0 & 1
\end{array}\right)=\left(\begin{array}{cc}
Q P_{+} & 0 \\
0 & 1
\end{array}\right)
$$

where

$$
Q:=\left(S P_{+}+P_{0}\right)^{-1 / 2}
$$


Hence, it suffices to prove the continuity of the operator $Q_{+} P_{+}$in $L_{\rho}^{2}$, which means the continuity of operator

$$
\langle x\rangle^{\rho} Q P_{+}\langle x\rangle^{-\rho}: L^{2}(\mathbb{R}) \longrightarrow L^{2}(\mathbb{R}) .
$$

To prove this continuity, we note that $Q$ is a PDO of the class $H G_{1}^{-1,-1}$, see Definition 25.2 in [40]. This fact follows from [12, Theorem 29.1.9] and also by an extension of [40, Theorem 11.2] to PDOs with nonempty continuous spectrum. It is important that operator $Q$ is a PDO with the main symbol $\xi^{2}$, and

$$
\xi^{2} \notin(-\infty, 0], \quad \xi \neq 0 ; \quad \sigma\left(S_{+}\right) \cap(-\infty, 0]=\emptyset
$$

by (4.12). Hence, conditions (10.1) and (10.2) of [40] hold.

Now the continuity (6.12) follows by the Theorem of Composition of the PDO.

Lemma 6.2 with $\rho=-s$ and Lemma 5.1 imply (cf. (5.4)) that

$$
a_{\omega}:=G h_{\omega} \in L_{-s}^{2} \otimes \mathbb{C}^{2}, \quad s>1 .
$$

Now we can prove the following lemma.

Lemma 6.3. $a_{\omega}$ are the generalized eigenfunctions of $A$ corresponding to the eigenvalues $-i \omega$.

Proof. Formulas (6.4) and (6.6) imply that

$$
X^{c}(t)=\int_{\sigma_{c}} e^{-i \omega t} C(\omega) a_{\omega} d \omega, \quad A X^{c}(t)=\int_{\sigma_{c}} e^{-i \omega t} \omega C(\omega) a_{\omega} d \omega,
$$

for $X^{c}(0) \in \mathcal{W}$ by definition (6.13), Lemma 6.1 and the last corollary with $\rho=$ $-s<-1$. These identities mean that $a_{\omega}$ are the generalized eigenfunctions in the sense of [36, (80b)].

Finally, the main result of our paper is the following.

Theorem 6.4. Let condition (4.14) hold, $X(0) \in \mathcal{V}$ and $s>1$. Then the eigenfunction expansion (5.6) holds in the following sense (cf. (5.13)):

$$
\left\|X^{c}(t)-\int_{m \leq|\omega| \leq M} e^{-i \omega t} C(\omega) a_{\omega} d \omega\right\|_{\mathcal{V}} \longrightarrow 0, \quad M \rightarrow \infty,
$$

where the integral converges in $L_{-s}^{2} \otimes \mathbb{C}^{2}$, and hence a.e. as in (6.1). 
Proof. Formulas (6.3) and (6.13) imply that

$$
\begin{aligned}
& X^{c}(t)-\int_{m \leq|\omega| \leq M} e^{-i \omega t} C(\omega) a_{\omega} d \omega \\
& =G\left[Z^{c}(t)-\int_{m \leq|\omega| \leq M} e^{-i \omega t} C(\omega) h_{\omega} d \omega\right] .
\end{aligned}
$$

Therefore, (6.15) follows from (5.13), because the Green operator $G: X \rightarrow \mathcal{V}$ is continuous.

\section{Symplectic normalization}

Now let us renormalize $h_{\omega}$ as follows:

$$
\left\langle h_{\omega}, h_{\omega^{\prime}}\right\rangle=|\omega| \delta\left(\omega-\omega^{\prime}\right), \quad \omega, \omega^{\prime} \in \sigma_{c} .
$$

This means that

$$
\left\langle Z_{1}, Z_{2}\right\rangle=\int_{m \leq|\omega| \leq M}|\omega| C_{1}(\omega) \overline{C_{2}(\omega)} d \omega
$$

for

$$
Z_{k}=\int_{m \leq|\omega| \leq M} C_{k}(\omega) h_{\omega} d \omega \in X,
$$

similarly to (5.10). We will express these formulas in terms of

$$
X_{k}:=G Z_{k} \in \mathcal{V}
$$

and the eigenfunctions

$$
a_{\omega}:=G h_{\omega}
$$

First,

$$
X_{k}=\int_{m \leq|\omega| \leq M} C_{k}(\omega) a_{\omega} d \omega
$$

by Lemma 6.2. Further, $Z_{k} \in R$, and so (5.12), (7.2) imply that

$$
\left\langle H_{R}^{-1} Z_{1}, Z_{2}\right\rangle=\int_{m \leq|\omega| \leq M} \operatorname{sgn} \omega C_{1}(\omega) \overline{C_{2}(\omega)} d \omega .
$$

On the other hand, this scalar product can be expressed in $X_{k}$. 
Lemma 7.1. Let $Z_{1}, Z_{2}$ be defined as in (7.2). Then

$$
\left\langle H_{R}^{-1} Z_{1}, Z_{2}\right\rangle=-i\left\langle X_{1}, J X_{2}\right\rangle .
$$

Proof. First, $Z_{1}, Z_{2} \in R \subset \mathcal{R}$, and hence,

$$
\Pi_{\mathcal{R}} Z_{k}=Z_{k}
$$

Now (6.10) implies (7.5)

$$
\begin{aligned}
\left\langle X_{1}, J X_{2}\right\rangle & =\left\langle G Z_{1}, J G Z_{2}\right\rangle \\
& =\left\langle\Lambda_{\mathcal{R}}^{-1} \Pi_{\mathcal{R}} Z_{1}, J \Lambda_{\mathcal{R}}^{-1} \Pi_{\mathcal{R}} Z_{2}\right\rangle \\
& =-\left\langle\Lambda_{\mathcal{R}}^{-1} \Pi_{\mathcal{R}} J \Lambda_{\mathcal{R}}^{-1} Z_{1}, Z_{2}\right\rangle \\
& =i\left\langle H_{R}^{-1} Z_{1}, Z_{2}\right\rangle,
\end{aligned}
$$

since

$$
\begin{aligned}
\Lambda_{\mathcal{R}}^{-1} \Pi_{\mathcal{R}} J \Lambda_{\mathcal{R}}^{-1} & =\left(\begin{array}{cc}
S_{+}^{-1 / 2} P_{+} & 0 \\
0 & 1
\end{array}\right)\left(\begin{array}{cc}
0 & 1 \\
-1 & 0
\end{array}\right)\left(\begin{array}{cc}
S_{+}^{-1 / 2} & 0 \\
0 & 1
\end{array}\right) \\
& =\left(\begin{array}{cc}
0 & S_{+}^{-1 / 2} \\
-S_{+}^{-1 / 2} & 0
\end{array}\right) \\
& =-i H_{R}^{-1}
\end{aligned}
$$

by the first formula of (4.11) and by (6.11) and (6.8).

Using this lemma and (7.4), we get

$$
-i\left\langle X_{1}, J X_{2}\right\rangle=\int_{m \leq|\omega| \leq M} \operatorname{sgn} \omega C_{1}(\omega) \overline{C_{2}(\omega)} d \omega .
$$

By definition, (7.3) and (7.6) mean that

$$
\left\langle a_{\omega}, J a_{\omega^{\prime}}\right\rangle=i \operatorname{sgn} \omega \delta\left(\omega-\omega^{\prime}\right), \quad \omega, \omega^{\prime} \in \sigma_{c} .
$$

Now expansion (5.6) coincides with [5, (2.1.13)], thereby justifying our calculation of the Fermi Golden Rule for all solutions without the antisymmetry condition imposed in [22]. 


\section{A. Examples}

Let us show that conditions (1.4), (2.12), and (3.1) hold for elliptic pseudo-differential operators

$$
P \psi(x)=\int e^{-i x \xi} P(x, \xi) \hat{\psi}(\xi) d \xi,
$$

which are the main objects of the theory. We will use the classes $\mathcal{S}^{m}$ of PDO similar to the ones introduced in [11].

Definition A.1. i) $P \in \mathcal{S}^{m}$ if, for any multiindices $\alpha, \beta$,

$$
\sup _{x \in \mathbb{R}^{n}}\left|(1+|x|)^{N} \partial_{\xi}^{\alpha} \partial_{x}^{\beta} P(x, \xi)\right| \leq C_{\alpha \beta N}(1+|\xi|)^{m-|\alpha|}, \quad \xi \in \mathbb{R}^{n}
$$

with $N=0$ for $\beta=0$ and any $N>0$ for $\beta \neq 0$.

ii) $P \in \mathcal{S}_{0}^{m}$ if (A.2) holds for any multiindices $\alpha, \beta$ and all $N>0$.

iii) $P \in \mathcal{S}^{m}$ is elliptic of order $m$ if $P=P_{m}+R$, where $P_{m} \in \mathcal{S}^{m}$ and

$$
\left|P_{m}(x, \xi)\right| \geq C(1+|\xi|)^{m}, \quad x, \xi \in \mathbb{R}^{n},
$$

while $R \in \mathcal{S}_{0}^{\mu}$ with $\mu<m$.

Let $\mathcal{H}^{s}=\mathcal{H}^{s}\left(\mathbb{R}^{n}\right)$ denote the Sobolev spaces, and $X=L^{2}\left(\mathbb{R}^{n}\right)$. Any operator $P \in \mathcal{S}^{m}$ is continuous $H^{s} \rightarrow H^{s-m}$ for $s \in \mathbb{R}$, see Theorem 3.1 of [11].

Lemma A.2. Let $B \in \mathcal{S}^{m}$ be an elliptic PDO of order $m$ which is symmetric on $C_{0}^{\infty}\left(\mathbb{R}^{n}\right)$, and let $J \in \mathcal{S}^{0}$ be an elliptic PDO of order 0 which is antisymmetric on $C_{0}^{\infty}\left(\mathbb{R}^{n}\right)$. Then

i) $B$ (respectively, $J$ ) is selfadjoint (respectively, skew selfadjoint) operator with domain

$$
D(B)=\mathcal{H}^{m}, \quad D(J)=X ;
$$

ii) condition (1.4) holds;

iii) condition (2.12) holds;

iv) condition (3.1) holds.

Proof. i) The Fredholm theory of elliptic PDO on $\mathbb{R}^{n}$ [40, Section 25.4] implies that $B \psi \in X$ if and only if $\psi \in \mathcal{H}^{m}$, and the same is true for $B^{*}$. Hence, $D\left(B^{*}\right)=$ $D(B)$, and therefore, $B^{*}=B$. Similarly, $J^{*}=-J$. 
ii) The Fredholm theory of elliptic PDOs on $\mathbb{R}^{n}$ implies that the space

$$
\mathcal{K}:=\operatorname{Ker} B
$$

is finite dimensional and $\mathcal{K} \subset \mathcal{H}^{s}$ for any $s \in \mathbb{R}$. Hence, (1.4) holds.

iii) The operator

$$
B_{+}:=B+\Pi_{\mathcal{K}}
$$

and its main symbol $B_{+}^{m}(x, \xi)$ satisfy

$$
B_{+}^{m}(x, \xi) \notin(-\infty, 0], \quad \xi \neq 0 ; \quad \sigma\left(B_{+}\right) \cap(-\infty, 0]=\emptyset
$$

by (1.3). Therefore, conditions (10.1) and (10.2) of [40] hold for $B_{+}$, and hence,

$$
\Lambda_{+}:=\sqrt{B_{+}} \geq 0
$$

is also an elliptic PDO of class $\mathcal{S}^{m / 2}$. This follows similarly to Theorem 29.1.9 of [12] and also by an extension of Theorem 11.2 of [40] to PDO with nonempty continuous spectrum. Finally,

$$
\Lambda_{+}=\Lambda+\Pi_{\mathcal{K}}
$$

Therefore, $\mathcal{V}=\mathcal{H}^{m / 2}$, and hence (2.12) holds, inasmuch as $J \in \mathcal{S}^{0}$.

iv) The operator $H$ is elliptic PDO of class $\mathcal{S}^{m}$ by the theorem of composition. It is obviously symmetric on $C_{0}^{\infty}\left(\mathbb{R}^{n}\right)$, and hence $H$ is selfadjoint on the domain $\mathcal{H}^{m}$ by the argument above. Thus (3.1) is established.

Remark A.3. i) An example of elliptic operators $B \in \mathcal{S}^{2}$ and $J \in \mathcal{S}^{0}$ satisfying all conditions (1.1), (1.3), (1.4), (2.12), and (3.1) is provided in Lemma 4.1.

ii) In the framework of Lemma A.2 we should take $m \geq 0$ to keep condition (1.3).

iii) The last condition of (1.1) implies that the order of $J$ should be zero.

\section{References}

[1] S. Agmon, Spectral properties of Schrödinger operators and scattering theory. Ann. Scuola Norm. Sup. Pisa Cl. Sci. (4) 2 (1975), no. 2, 151-218. MR 0397194 Zbl 0315.47007

[2] T. Ya. Azizov, I. S. Iokhvidov, Linear operators in spaces with an indefinite metric. Nauka, Moscow, 1986. In Russian. English trasl. by E. R. Dawson. Pure and Applied Mathematics (New York). John Wiley \& Sons, Chichester, 1989. MR 1033489 MR 0863885 (transl.) Zbl 0714.47028 Zbl 0607.47031 (trasl.) 
[3] V. S. Buslaev and G. S. Perelman, Scattering for the nonlinear Schrödinger equation: states close to a soliton. Algebra i Analiz 4 (1992), no. 6, 63-102. In Russian. English transl., St. Petersburg Math. J. 4 (1993), no. 6, 1111-1142. MR 1199635 Zbl 0853.35112

[4] V. S. Buslaev and G. S.+ Perelman, On the stability of solitary waves for nonlinear Schrödinger equations. Nonlinear evolution equations. In N. N. Uraltseva (ed.), Nonlinear evolution equations. Translated from the original Russian manuscripts by A. B. Sossinsky. American Mathematical Society Translations, Series 2, 164. Advances in the Mathematical Sciences, 22. American Mathematical Society, Providence, R.I., 1995, 75-98. MR 1334139 MR 1334135 (collection) Zbl 0841.35108 Zbl 0824.00037 (collection)

[5] V. S. Buslaev and C. Sulem, On asymptotic stability of solitary waves for nonlinear Schrödinger equations. Ann. Inst. H. Poincaré Anal. Non Linéaire 20 (2003), no. 3, 419-475. MR 1972870 Zbl 1028.35139

[6] T. Cazenave and A. Haraux, An introduction to semilinear evolution equations. Translated from the 1990 French original by Y. Martel and revised by the authors. Oxford Lecture Series in Mathematics and its Applications, 13. The Clarendon Press, Oxford University Press, New York, 1998. MR 1691574 Zbl 0926.35049

[7] S. Cuccagna, D. Pelinovsky, and V. Vougalter, Spectra of positive and negative energies in the linearized NLS problem. Comm. Pure Appl. Math. 58 (2005), no. 1, 1-29. MR 2094265 Zbl 1064.35181

[8] M. B. Erdogan and W. Schlag, Dispersive estimates for Schrödinger operators in the presence of a resonance and/or an eigenvalue at zero energy in dimension three. II. J. Anal. Math. 99 (2006), 199-248. MR 2279551 Zbl 1146.35324

[9] C. Gérard, Scattering theory for Klein-Gordon equations with non-positive energy. Ann. Henri Poincaré 13 (2012), no. 4, 883-941. MR 2913626 Zbl 1253.81049

[10] I. C. Gohberg and M. G. Krein, Theory and applications of Volterra operators in Hilbert space. Nauka, Moscow, 1965. In Russian. English transl by A. Feinstein. Translations of Mathematical Monographs, 24. American Mathematical Society, Providence, R.I., 1969. MR 0264447 Zbl 0194.43804

[11] V. V. Grushin, Pseudodifferential operators on $\mathbb{R}^{n}$ with bounded symbols. Funkcional. Anal. i Priložen 4 (1970), no. 3, 37-50. In Russian. English transl., Funct. Anal. Appl. 4 (1970), 202-212. MR 0270214 Zbl 0223.35084

[12] L. Hörmander, The analysis of linear partial differential operators. IV. Fourier integral operators. Classics in Mathematics. Springer, Berlin, 2009. MR 2512677 Zbl 1178.35003

[13] V. Imaykin, A. I. Komech, and H. Spohn, Scattering asymptotics for a charged particle coupled to the Maxwell field. J. Math. Phys. 52 (2011), no. 4, Article id. 042701, 33 pp. MR 2964169

[14] I. S. Iohvidov, M. G. Krein, and H. Langer, Introduction to the spectral theory of operators in spaces with an indefinite metric. Mathematical Research, Vol. 9, AkademieVerlag, Berlin, 1982. MR 0691137 Zbl 0506.47022 
[15] P. Jonas, On the functional calculus and the spectral function for definitizable operators in Krein space. Beiträge Anal. 16 (1981), 121-135. MR 0663475 Zbl 0556.47019

[16] P. Jonas, On a class of unitary operators in Krein space. In R. G. Douglas, C. M. Pearcy, B. Szókefalvi-Nagy, F.-H. Vasilescu, D. Voiculescu, and Gr. Arsene, Advances in invariant subspaces and other results of operator theory. Proceedings of the $9^{\text {th }}$ International Conference on Operator Theory held in Timişoara and in Herculane, June 4-14, 1984. Operator Theory: Advances and Applications, 17. Birkhäuser, Basel etc., 1986, 151-172. MR 0901067 MR 0901055 (collection) Zbl 0617.47026 Zbl 0579.00011 (collection)

[17] P. Jonas, On a class of selfadjoint operators in Krein space and their compact perturbations. Integral Equations Operator Theory 11 (1988), no. 3, 351-384. MR 0938849 Zbl 0651.47020

[18] P. Jonas, On the spectral theory of operators associated with perturbed Klein-Gordon and wave type equations. J. Operator Theory 29 (1993), no. 2, 207-224. MR 1284880 Zbl 0818.35107

[19] P. Jonas, On bounded perturbations of operators of Klein-Gordon type. Glas. Mat. Ser. III 35(55) (2000), no. 1, 59-74. Dedicated to the memory of B. Najman. MR 1783232 Zbl 1017.47030

[20] T. Kako, Spectral and scattering theory for the $J$-selfadjoint operators associated with the perturbed Klein-Gordon type equations. J. Fac. Sci. Univ. Tokyo Sec. IA Math $\mathbf{2 3}$ (1976), no. 1, 199-221. MR 0412634 Zbl 0367.47026

[21] A. I. Komech, E. A. Kopylova, and H. Spohn, Scattering of solitons for Dirac equation coupled to a particle. J. Math. Anal. Appl. 383 (2011), no. 2, 265-290. MR 2812381 Zbl 1229.35228

[22] E. A. Kopylova and A. I. Komech, On asymptotic stability of kink for relativistic Ginzburg-Landau equations. Arch. Rational. Mech. Anal. 202 (2011), no. 2, 213-245. MR 2835867 Zbl 1256.35146

[23] E. A. Kopylova and A. I. Komech, On asymptotic stability of moving kink for relativistic Ginzburg-Landau equation. Comm. Math. Physics 302 (2011), no. 1, 225-252. MR 2770013 Zbl 1209.35134

[24] A. Komech and E. A. Kopylova, Dispersion decay and scattering theory. John Wiley \& Sons, Hoboken, N.J., 2012. MR 3015024 Zbl 06042927

[25] E. Kopylova, Dispersion estimates for the Schrödinger and Klein-Gordon equations. Uspekhi Mat. Nauk 65 (2010), no. 1(391), 97-144. In Russian. English transl., Russian Math. Surveys 65 (2010), no. 1, 95-142. MR 2655244 Zbl 1201.35061

[26] A. Komech and E. A. Kopylova, On eigenfunction expansion of solutions to the Hamilton equations. J. Stat. Phys. 154 (2014), no. 1-2, 503-521. MR 3162551 Zbl 1300.34195

[27] M. G. Krein, H. K. Langer, The spectral function of a selfadjoint operator in a space with indefinite metric. Dokl. Akad. Nauk SSSR 152 (1963), 39-42. In Russian. English transl., Sov. Math. Dokl. 4 (1963), 1236-1239. MR 0165366 Zbl 0131.12604 
[28] M. G. Krein, Yu. Shmul'yan, J-polar representations of plus-operators. Mat. Issled. 1 (1966), no. 2, 172-210. In Russian. MR 0208373

[29] H. Langer, Spectral functions of definitizable operators in Krein spaces. In D. Butković, H. Kraljević, and S. Kurepa (eds.), Functional analysis. Proceedings of a Conference held in Dubrovnik, November 2-14, 1981. Lecture Notes in Mathematics, 948. Springer, Berlin etc., 1982, 1-46. MR 0672791 MR 0672790 (collection) Zbl 0511.47023 Zbl 0482.00016 (collection)

[30] H. Langer and B. Najman, Perturbation theory for definitizable operators in Krein spaces. J. Operator Theory 9 (1983), no. 2, 297-317. MR 0703812 Zbl 0516.47021

[31] H. Langer, B. Najman, and C. Tretter, Spectral theory of the Klein-Gordon equation in Krein spaces. Proc. Edinb. Math. Soc. (2) 51 (2008), no. 3, 711-750. MR 2465932 Zbl 1152.81020

[32] H. Langer, B. Najman, and C. Tretter, Spectral theory of the Klein-Gordon equation in Pontryagin spaces. Comm. Math. Phys. 267 (2006), no. 1, 159-180. MR 2238908 Zbl 1114.47038

[33] H. Langer and C. Tretter, Variational principles for eigenvalues of the Klein-Gordon equation. J. Math. Phys. 47 (2006), no. 10, Article id. 103506, 18 pp. MR 2268872 Zbl 1112.78008

[34] M. Reed and B. Simon, Methods of modern mathematical physics I. Functional analysis. Academic Press, New York etc., 1972. MR 0493419 Zbl 0242.46001

[35] M. Reed and B. Simon, Methods of modern mathematical physics. II. Fourier analysis, self-adjointness. Academic Press, New York etc., 1975. MR 0493420

Zbl 0308.47002

[36] M. Reed and B. Simon, Methods of modern mathematical physics. III. Scattering theory. Academic Press, New York etc., 1979. MR 0529429 Zbl 0405.47007

[37] W. Rudin, Functional analysis. $2^{\text {nd }}$ ed. International Series in Pure and Applied Mathematics. McGraw-Hill, New York, 1991. MR 1157815 Zbl 0867.46001

[38] J. J. Sakurai, Advanced quantum mechanics. Addison-Wesley Series in Advanced Physics. Addison-Wesley, Reading, MA, etc., 1967. Zbl 0158.45604

[39] W. Schlag, Dispersive estimates for Schrödinger operators: a survey. In J. Bourgain, C. E. Kenig, and S. Klainerman (eds.), Mathematical aspects of nonlinear dispersive equations. Papers from the CMI/IAS Workshop on Mathematical Aspects of Nonlinear PDEs held in Princeton, N.J., 2004. Annals of Mathematics Studies, 163. Princeton University Press, Princeton, N.J., 2007, 255-285. MR 2333215

MR 2332225 (collection) Zbl 1143.35001 Zbl 1113.35005 (collection)

[40] M. A. Shubin, Pseudodifferential operators and spectral theory. Nauka, Moscow, 1978. In Russian. English transl. by S. I. Andersson. Springer Series in Soviet Mathematics. Springer, Berlin etc., 1987. MR 0509034 MR 0883081 (transl.)

Zbl 0451.47064 Zbl 0616.47040 (transl.)

[41] R. T. Seeley, Complex powers of an elliptic operator. Proc. Sympos. Pure Math 10 (1967), 288-307. MR 0237943 Zbl 0159.15504 
[42] I. M. Sigal, Nonlinear wave and Schrödinger equations. I. Instability of periodic and quasiperiodic solutions. Comm. Math. Phys. 153 (1993), no. 2, 297-320. MR 1218303 Zbl 0780.35106

[43] K. Yosida, Functional analysis. $6^{\text {th }}$ ed. Grundlehren der Mathematischen Wissenschaften, 123. Springer, Berlin etc., 1980. MR 0617913 MR 0435.46002

Received September 9, 2013

Alexander Komech, Faculty of Mathematics of Vienna University,

Oskar-Morgenstern-Platz 1, 1090 Wien, Austria

Institute for Information Transmission Problems

of the Russian Academy of Sciences (Kharkevich Institute),

Bolshoy Karetny per. 19, build.1, Moscow 127051 Russia

e-mail: alexander.komech@univie.ac.at

Elena Kopylova, Faculty of Mathematics of Vienna University,

Oskar-Morgenstern-Platz 1, 1090 Wien, Austria

Institute for Information Transmission Problems

of the Russian Academy of Sciences (Kharkevich Institute),

Bolshoy Karetny per. 19, build.1, Moscow 127051 Russia

e-mail: elena.kopylova@univie.ac.at 